\title{
EVOLUÇÃO E IMPLEMENTAÇÃO DOS PLANOS DE MANEJO EM PARQUES NACIONAIS NO ESTADO DO RIO DE JANEIRO ${ }^{1}$
}

\author{
Rodrigo Medeiros² e Gustavo Simas Pereira ${ }^{3}$
}

\begin{abstract}
RESUMO - O plano de manejo é um documento técnico mediante o qual se estabelece o zoneamento e as normas que devem presidir o uso da área de uma unidade de conservação e o manejo dos recursos naturais, inclusive a implantação das estruturas físicas necessárias à gestão. Eles foram legalmente instituídos no Brasil em 1979 com o objetivo de adequar e orientar o manejo ecológico dos parques nacionais, até então em sua maioria criados apenas no papel, apontando a necessidade de realizar o zoneamento como base do ordenamento e gestão do território protegido. Contudo, o processo de elaboração e implementação desse documento foi extremamente variado e sofreu uma série de modificações ao longo do tempo visando seu aperfeiçoamento. O objetivo deste trabalho foi analisar a evolução do plano de manejo no contexto da gestão dos parques nacionais no país analisando como caso concreto a sua implementação no estado do Rio de Janeiro. A elaboração de planos de manejo para parques no Brasil foi marcada pela existência de três referências metodológicas distintas o que se refletiu na variedade de planos identificados para os cinco parques nacionais analisados neste trabalho (PARNAs do Itatiaia, da Serra dos Órgãos, da Tijuca, da Serra da Bocaina e da Restinga da Jurubatiba). Como padrão geral os planos de manejo não foram elaborados, nem revisados dentro dos prazos e periodicidade estabelecida pela legislação, o que denota uma dificuldade na implementação desse instrumento mesmo quase trinta anos após a sua instituição.
\end{abstract}

Palavras-Chaves: Plano de manejo, Parque nacional e Unidade de conservação

\section{EVOLUTION AND IMPLEMENTATION OF MANAGEMENT PLANS IN NATIONAL PARKS IN THE STATE OF RIO DE JANEIRO.}

\begin{abstract}
Management plan is a technical document that establish zoning and rules that will define the protect area usage and its natural resources management. In 1979 they were legally established in Brazil aiming to adapt and orientate the ecological handling of national parks, which are still not fully implemented. This indicates the necessity of carrying out the zoning as a base to the protected territory planning. However, preparation and implementation process of this document was extremely varied and suffered several modifications aiming its improvement. The objective of this work was to analyze the evolution of management plans in the context of national parks management, analyzing as a concrete case its implementation in the state of Rio de Janeiro, Brazil. The preparation of management plans for Brazilian parks was marked by the existence of three different methodological references that was reflected in the variety of plans identified for five national parks studied here (Itatiaia NP, Serra dos Órgãos NP, Tijuca NP, Serra da Bocaina NP and Restinga da Jurubatiba NP). As a general pattern, the plans were neither prepared nor revised with the periodicity established by the legislation. This shows a difficulty in the implementation of this instrument even almost thirty years after its institution.
\end{abstract}

Keywords: Management plan, National park and Protected area.

\footnotetext{
${ }^{1}$ Recebido em 22.12.2008 e aceito para publicação em 16.12.2010.

${ }^{2}$ Laboratório de Gestão Ambiental, Instituto de Florestas, Departamento de Ciências Ambientais, UFRRJ. . E-mail: <medeiros@ufrrj.br> e<gustavosimas@gmail.com>..
} 


\section{INTRODUÇÃO}

As áreas protegidas representam no mundo contemporâneo importante estratégia de proteção dos recursos naturais e culturas associadas (LOVEJOY, 2006). A sua implementação, sem os instrumentos adequados de planejamento e gestão, compromete as finalidades para a qual ela foi criada (ERVIN, 2004). O estabelecimento de sistemas de avaliação da efetividade e da abrangência das áreas protegidas tem sido sistematicamente desenvolvido em todo o mundo, como forma de melhorar esse processo (HOCKINGS, 2003; PARRISH et al., 2003; BROOKS et al., 2003).

No Brasil, cinco diferentes tipologias de áreas protegidas estão previstas na legislação: as unidades de conservação; as áreas de reconhecimento internacional; as terras indígenas; as reservas legais; e as áreas de preservação permanente (MEDEIROS e GARAY, 2006). Dado o contexto histórico, as unidades de conservação representam a tipologia mais antiga de área protegida. A sua proposição data do final do século XIX (PÁDUA, 2003), porém sua materialização em nível federal só ocorreu em 1937, com a criação do Parque Nacional do Itatiaia (MEDEIROS et al., 2004; RYLANDS e BRANDON, 2005; MEDEIROS, 2006). As áreas protegidas são compostas atualmente por um grupo de 13 categorias de manejo distintas, nas quais estão incluídos os parques nacionais.

Os parques nacionais (PARNA) foram criados a partir da instituição do Código Florestal de 1934 (Decreto 23.793), que estabeleceu em seu artigo nono que (sic) "os parques nacionaes, estaduaes ou municipaes, constituem monumentos publicos naturaes, que perpetuam em sua composição floristica primitiva, trechos do paiz, que, por circumstancias peculiares, o merecem” (BRASIL, 1934, p. 3). Nos anos que sucederam a criação do primeiro parque, novas unidades foram estabelecidas. Um novo Código Florestal em 1965 consagrou os parques como principal categoria para fins de proteção integral e, até o final dos anos de 1970, 20 parques nacionais já haviam sido criados no país sem que sua prática houvesse sido devidamente regulamentada (MEDEIROS, 2006).

A regulamentação dos parques nacionais só ocorreu em 21 de setembro de 1979, através do Decreto n ${ }^{\circ}$ 84.017, 42 anos após a criação do PARNA do Itatiaia. Esse Decreto definiu melhor as bases para a criação e gestão dessas áreas. Um dos aspectos mais relevantes desse Decreto foi a instituição, em seu artigo quinto, do plano de manejo definido como "um projeto dinâmico que, utilizando técnicas de planejamento ecológico, determine o zoneamento de um Parque Nacional, caracterizando cada uma das suas zonas e propondo seu desenvolvimento físico, de acordo com suas finalidades” (BRASIL, 1979, p. 2). Seu objetivo era adequar e orientar o manejo ecológico dos parques nacionais, até então em sua maioria criados apenas no papel, apontando a necessidade de realizar o zoneamento como base do ordenamento e gestão do território protegido. A lógica era estabelecer zonas diferenciadas onde os atributos e vocações da área iriam determinar o conjunto de intervenções e atividades permitidas (como exemplo as zonas intangível, primitiva, de uso extensivo, de usos intensivo, histórico-cultural e de recuperação, entre outras).

O estabelecimento dos planos de manejo na legislação brasileira, contudo, tampouco foi iniciativa original. Ele apenas consolidou uma prática de planejamento para gestão de parques iniciada na tradição norteamericana e foi amplamente difundida para a América Latina a partir dos anos de 1960. Até o final dessa década, Venezuela, Chile e Peru já haviam estabelecido planos de manejo para seus parques. Na década seguinte, pelo menos 55 parques em toda a América Latina seguiram os mesmos passos (MILLER, 1989; DOUROJEANNI, 2005). O processo básico de elaboração era guiado por uma série de estudos e recomendações feitas por instituições ligadas ao tema, como a União Mundial para a Conservação da Natureza/UICN (HOROWITZ, 2000) e o Departamento de Florestas da Organização das Nações Unidas para Agricultura e Alimentação (FAO) (MILLER, 1989).

No Brasil, essa prática mobilizou alguns setores envolvidos com o movimento parquista, incluindo o Instituto Brasileiro de Desenvolvimento Florestal (IBDF), órgão responsável pela gestão de parques no Brasil que se mobiliza com o apoio de especialistas estrangeiros para a realização dos primeiros planos de manejo. Como consequência, em 1976 foram concluídos os planos de manejo do Parque Nacional de Sete Cidades e do Parque Nacional de Brasília (WETTERBERG, 2004; DOUROJEANNI, 2005). Dessa forma, a obrigatoriedade estabelecida pela legislação em 1979 apenas legitimou e consagrou uma prática iniciada no país poucos anos antes. 
A instituição do Sistema Nacional de Unidades de Conservação da Natureza (SNUC), em 2000, consolidou o plano de manejo como ferramenta central do processo de gestão para todas as categorias de unidades de conservação (BRASIL, 2000). Seu Artigo 27 estabeleceu que "todas as unidades de conservação devem dispor de um plano de manejo" e definiu a abrangência e os prazos de sua elaboração. Apesar da obrigatoriedade estabelecida pela lei, a elaboração e implementação dos planos de manejo no Brasil estiveram longe de ser uma realidade consolidada. Estima-se que apenas cerca de $15 \%$ do total de unidades de conservação no país possuam plano de manejo devidamente aprovado e atualizado. Entre os parques nacionais, esse número não atinge 20\% deles (IBAMA, 2008). Essa realidade revela ainda o crônico problema de planejamento na gestão dessas áreas, que se traduz em um dos desafios a serem superados pelos órgãos gestores.

No total, 64 Parques Nacionais foram criados no Brasil até novembro de 2008, abrangendo cerca de 23 milhões de hectares. Esse número representa aproximadamente $10 \%$ do total de unidades de conservação no país e equivale a 2,7\% do território brasileiro (IBAMA, 2008). Os parques representam a categoria mais representativa entre aquelas de proteção integral e a segunda com maior extensão entre todas as unidades de conservação. Cinco desses parques estão localizados no Estado do Rio de Janeiro, sendo dois deles os mais antigos estabelecidos no Brasil (PARNA do Itatiaia, criado em 1937; e PARNA da Serra dos Órgãos, criado em 1939). Apesar disso, poucas informações são disponíveis sobre o estado de implementação de seus planos de manejo, bem como sobre sua efetividade no processo de gestão.

O objetivo deste trabalho foi analisar a evolução do plano de manejo no contexto da gestão de parques nacionais no país, analisando como caso concreto a sua implementação no Estado do Rio de Janeiro.

\section{MATERIAL E MÉTODOS}

Para a realização deste estudo, foram selecionados os cinco parques nacionais (PARNA) localizados no Estado do Rio de Janeiro: o PARNA do Itatiaia, o PARNA da Serra dos Órgãos, o PARNA da Tijuca, o PARNA da Serra da Bocaina e o PARNA da Restinga da Jurubatiba. Os parques nacionais foram escolhidos por pertencerem à categoria de área protegida mais antiga e serem os primeiros a apresentar a obrigatoriedade do plano de manejo. Dessa forma, pressupõe-se que essas fossem condições favoráveis para que os planos de manejo estivessem em melhor situação de criação e implementação.

A caracterização de cada um dos parques estudados foi feita a partir do acesso às informações disponíveis no Banco de Dados sobre Áreas Protegidas (SIUCWEB) do IBAMA (IBAMA, 2008), no Cadastro Nacional de Unidades de Conservação (CNUC), da Diretoria de Áreas Protegidas do Ministério do Meio Ambiente (MMA, 2008) e em documentos técnicos que fazem parte do acervo dos parques. Toda a legislação relacionada aos parques estudados também foi consultada e analisada, a partir do acesso realizado na Base da Legislação Federal da Casa Civil da Presidência da República (BRASIL, 2008).

Uma cópia impressa dos Planos de Manejo dos parques foi obtida na gestão deles, e uma análise qualitativa do seu conteúdo, conforme descrito por Bardin (1977), foi realizada visando buscar informações relativas ao seu processo de elaboração, aprovação, implementação e revisão.

Entrevistas com os gestores dos parques estudados também foram realizadas com o intuito de obter informações sobre o plano de manejo, seu uso no contexto da gestão do parque e o andamento do processo de elaboração ou revisão deles.

A descrição da evolução do plano de manejo no contexto dos parques nacionais no Brasil foi feita com base na análise comparativa de toda a legislação federal pertinente e da bibliografia disponível sobre o tema, além de documentos técnicos como os roteiros metodológicos oficiais para elaboração de plano de manejo (IBAMA, 1996; 2002).

\section{RESULTADOS E DISCUSSÃO}

Na Tabela 1, apresentam-se as informações de caracterização dos cinco parques nacionais estudados neste trabalho. Desse total, a maioria (quatro) foi criada antes da regulamentação que instituiu a obrigatoriedade do Plano de Manejo em 1979 (Decreto n ${ }^{\circ}$ 84.017) e um ainda antes do estabelecimento do SNUC em 2000 (Lei n ${ }^{\circ}$ 9.985). Todos os parques possuíam pelo menos uma versão do plano de manejo devidamente elaborado e publicado.

O regulamento de parques nacionais, em 1979, definiu no Artigo 43 que o IBDF teria prazo de cinco anos para elaboração dos planos de manejo dos parques

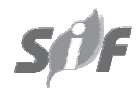

Revista Árvore, Viçosa-MG, v.35, n.2, p.279-288, 2011 
Tabela 1 - Caracterização geral dos Parques Nacionais no Estado do Rio de Janeiro.

Table 1 - General characterization of National Parks in the State of Rio de Janeiro.

\begin{tabular}{|c|c|c|c|c|c|c|}
\hline Nome & Data Criação & $\begin{array}{c}\text { Decreto } \\
\text { de Criação }\end{array}$ & $\begin{array}{l}\text { Área } \\
\text { (ha) }\end{array}$ & $\begin{array}{l}\text { Municípios } \\
\text { abrangidos }\end{array}$ & $\begin{array}{l}\text { Plano de } \\
\text { Manejo }\end{array}$ & $\begin{array}{c}\text { Situação do } \\
\text { Plano de Manejo }\end{array}$ \\
\hline $\begin{array}{l}\text { PARNA do } \\
\text { Itatiaia }\end{array}$ & $\begin{array}{c}14 \text { de junho de } \\
1937\end{array}$ & $1.713 / 37$ & 56.310 & $\begin{array}{c}\text { Alagoa (MG); Bocaina de } \\
\text { Minas (MG); Itamonte } \\
\text { (MG); Itatiaia (RJ); } \\
\text { Resende (RJ) }\end{array}$ & $\begin{array}{l}\text { Versão única } \\
\quad=1982\end{array}$ & $\begin{array}{l}\text { Em vigência e } \\
\text { iniciando a } \\
\text { revisão }\end{array}$ \\
\hline $\begin{array}{l}\text { PARNA da } \\
\text { Serra dos } \\
\text { Órgãos } \\
\end{array}$ & $\begin{array}{c}30 \text { de novembro } \\
\text { de } 1939\end{array}$ & $1.822 / 39$ & 21.054 & $\begin{array}{l}\text { Guapimirim (RJ); Magé } \\
\text { (RJ); Petrópolis (RJ); } \\
\text { Teresópolis (RJ) }\end{array}$ & $\begin{array}{c}\text { 1a versão }=1980 \\
\text { Revisão }=2008\end{array}$ & $\begin{array}{l}\text { Versão revisada } \\
\text { em vigência }\end{array}$ \\
\hline $\begin{array}{l}\text { PARNA da } \\
\text { Tijuca } \\
\end{array}$ & $\begin{array}{c}06 \text { de julho } \\
\text { de } 1961\end{array}$ & $50.923 / 61$ & 11.926 & Rio de Janeiro (RJ) & $\begin{array}{c}1 \text { a versão }=1981 \\
\text { Revisão }=2008\end{array}$ & $\begin{array}{c}\text { Versão revisada } \\
\text { em vigência }\end{array}$ \\
\hline $\begin{array}{l}\text { PARNA da } \\
\text { Serra da } \\
\text { Bocaina }\end{array}$ & $\begin{array}{c}04 \text { de fevereiro } \\
\text { de } 1971\end{array}$ & $68.172 / 71$ & 196.226 & $\begin{array}{c}\text { Angra dos Reis (RJ); } \\
\text { Parati (RJ); Areias (SP); } \\
\text { Bananal (SP); Cunha } \\
\text { (SP); São José do Barreiro } \\
\text { (SP); Ubatuba (SP) }\end{array}$ & $\begin{array}{l}\text { Versão única } \\
\quad=2002\end{array}$ & $\begin{array}{c}\text { Em vigência sem } \\
\text { previsão de início } \\
\text { de revisão }\end{array}$ \\
\hline $\begin{array}{l}\text { PARNA da } \\
\text { Restinga de } \\
\text { Jurubatiba }\end{array}$ & $\begin{array}{c}29 \text { de abril de } \\
1998\end{array}$ & $\begin{array}{c}\text { s/n de } \\
29 / 04 / 98\end{array}$ & 14.903 & $\begin{array}{l}\text { Carapebus (RJ); Macaé } \\
\text { (RJ);Quissamã (RJ) }\end{array}$ & $\begin{array}{l}\text { Versão única } \\
\quad=2008\end{array}$ & Em vigência \\
\hline
\end{tabular}

Fonte: Cadastro Nacional de Unidades de Conservação (MMA/DAP); IBAMA (Siucweb).

já criados. Para os novos parques, esse prazo seria indicado no próprio Decreto de criação (BRASIL, 1979). Dos quatro parques criados antes da regulamentação, apenas o PARNA da Serra da Bocaina não cumpriu esse prazo, tendo seu plano aprovado somente em 2002. Não foram encontrados registros, nem mesmo no texto do plano de manejo vigente, que expliquem a razão de esse parque, ao contrário dos outros, não ter sido contemplado pelo IBDF na elaboração do seu plano.

Os Planos de Manejo dos Parques Nacionais da Serra dos Órgãos (IBDF/FBCN, 1980), da Tijuca (IBDF/ FBCN, 1981) e do Itatiaia (IBDF/FBCN, 1982) foram todos elaborados, sucessivamente, a partir de parceria entre o IBDF e a Fundação Brasileira para Conservação da Natureza (FBCN). De maneira geral, a análise dos seus conteúdos revelou que eles apresentam a mesma estrutura de organização e foram concebidos seguindo as recomendações do Decreto nº 84.017/79.

O plano do Parque Nacional da Serra da Bocaina teve sua elaboração iniciada somente em 1996, já sob a gestão do IBAMA, através de um convênio firmado entre o Ministério do Meio Ambiente, a Diretoria de Ecossistemas (DIREC) do IBAMA e a Associação PróBocaina (IBAMA, 2002). O processo de elaboração contou com três equipes técnicas distintas, sob a coordenação da DIREC, resultando em documentos preliminares produzidos entre julho de 1997 e abril de 2000. Como consequência, o plano só foi efetivamente finalizado e publicado em 2002 (IBAMA, 2002).
O Parque Nacional da Restinga da Jurubatiba é o mais recente de todos os parques fluminenses, criado em 29 de abril de 1998 (BRASIL, 1998). Conforme estabelecido pelo Artigo sexto de seu Decreto de criação, o IBAMA teria o prazo máximo de cinco anos para a elaboração do plano de manejo. As mobilizações para sua elaboração tiveram início em 2000 com a contratação de consultoria técnica para auxiliar a DIREC/IBAMA nesse processo. Os trabalhos avançaram até meados de 2002, quando tiveram de ser interrompidos em função da mudança no roteiro metodológico de referência para planos de manejo (ICMBIO, 2008c). Os trabalhos foram retomados em 2003, e a conclusão e publicação do plano ocorreram em agosto de 2008, dentro do prazo estabelecido pelo Decreto, considerando-se a retomada dos trabalhos com base no novo roteiro.

Um traço comum entre todos os planos analisados neste estudo é que cada um deles, inclusive suas revisões, foi elaborado tendo como referência orientação técnica distinta. Isso demonstra claramente que a definição dos planos de manejo como instrumento de gestão de parques no Brasil não constituiu tarefa finalizada, mas sim um processo em que o aprendizado e amadurecimento dos órgãos gestores possibilitaram a revisão dos procedimentos e orientações técnicas mais adequadas à realidade nacional. Situação semelhante também foi observada por Souza e Martos (2008) ao analisarem o plano de manejo da FLONA de Ipanema. 
Nesse processo de construção dos planos de manejo, três fases distintas puderam ser identificadas. A primeira foi marcada pelo Decreto que estabeleceu a obrigatoriedade dos planos de manejo em 1979 e perdurou até 1996. Nesse período, a única referência oficial para a elaboração do plano foi o próprio Decreto de regulamentação dos parques nacionais de 1979 . O texto delimitou algumas informações que deveriam compor o documento, sobretudo com relação ao zoneamento. No entanto, o Decreto não constituiu orientação técnica específica e detalhada. O que fez que boa parte dos planos de manejo elaborados sob sua influência tenha se baseado em recomendações e metodologias adaptadas de outros países. Nesse contexto, os trabalhos desenvolvidos por Kenton Miller em parques na América Latina e as recomendações do Departamento de Florestas da Organização das Nações Unidas para Agricultura eAlimentação (FAO), em cooperação com o IBDF, foram as diretrizes mais frequentemente adotadas para a elaboração dos planos de manejo (MILLER, 1989; DOUROJEANNI, 2005). Uma consequência desse processo, como discutiu Dourojeanni (2005, p. 2), foi que "a elaboração de planos de manejo foi se diversificando, afastando-se progressivamente do modelo original e adotando esquemas diferentes em cada estado e em cada agência como, por exemplo, no caso dos planos de manejo das estações ecológicas, patrocinados pela antiga Secretária Especial de Meio Ambiente”.

Com a criação do IBAMA em 1989 e o reconhecimento de que a elaboração e aplicação dos planos de manejo de fato não atendiam plenamente aos objetivos esperados, buscaram-se aperfeiçoar e padronizar a sua confecção. Esse procedimento era essencial, já que alguns agentes de financiamento de unidades de conservação no país, como o Programa Nacional de Meio Ambiente (PNMA), condicionavam a liberação de recursos à elaboração de planos de manejo. Havia, assim, a necessidade de encontrar solução adequada, uma vez que com a ausência de planos de manejo elaborados ou revisados não se conheciam as prioridades para aplicação dos recursos financeiros (SANTOS, 2000). A alternativa encontrada foi a criação de instrumento mais simplificado de planejamento, chamado de Plano de Ação Emergencial (PAE). Em 1993, o IBAMA publicou o "Roteiro Metodológico para a Elaboração de Planos de Ação para Implementação e Gerenciamento de Unidades de Conservação de Uso Indireto” para orientar a elaboração do PAE (IBAMA,
1995). No período de dezembro de 1993 a outubro de 1995, no âmbito do PNMA foram priorizadas 28 unidades de conservação, que tiveram seus Planos de Ação Emergencial elaborados. Entre as áreas selecionadas estavam o Parque Nacional da Serra dos Órgãos e o Parque Nacional do Itatiaia (IBAMA, 1994ab). No mesmo ano, 1994, um PAE para o Parque Nacional da Tijuca também foi realizado, porém não foi aprovado pelo IBAMA (ICMBIO, 2008a).

O objetivo, no entanto, não era de que o PAE substituísse os planos de manejo. Pelo contrário, esperava-se que eles acabassem constituindo instrumento emergencial de suporte à gestão e que subsidiassem a confecção ou revisão dos planos de manejo das áreas beneficiadas. Dessa forma, uma nova orientação técnica foi elaborada pelo IBAMA em 1996, consolidada no "Roteiro Metodológico para o Planejamento de Unidades de Conservação de Uso Indireto” (IBAMA, 1996).

Em seu texto, esse novo roteiro que marca o início da segunda fase de planejamento dos parques nacionais reconhecia as dificuldades de elaboração e implementação dos planos de manejo e procurava:

Estabelecer uma metodologia mais flexível e dinâmica que permita iniciar o processo de planejamento em um maior número de unidades de conservação, proporcionando a estas um instrumento que irá progressivamente evoluindo em conhecimento e ações (IBAMA, 1996, p. 2).

A ideia principal era de que esse roteiro seria orientação temporária a ser implementada como parte de um projeto-piloto em quatro unidades de uso indireto, uma delas o Parque Nacional da Serra da Bocaina. A aplicação desse roteiro extrapolou a ideia inicial de quatro unidades, porém ele sobreviveu pouco tempo em parte, como foi apurado durante as entrevistas com gestores envolvidos na sua construção e aplicação, devido à sua complexidade e às dificuldades de elaboração. Como consequência, um novo roteiro foi elaborado e publicado em 2002 pelo IBAMA.

O "Roteiro metodológico de planejamento-Parques Nacionais, Reserva Biológica, Estação Ecológica” (IBAMA, 2002) marca o início da terceira fase na evolução do processo de elaboração de planos de manejo para parques nacionais no Brasil. Ele apontou a necessidade de corrigir falhas detectadas no roteiro de 1996 e buscou absorver elementos de outras metodologias que se mostraram úteis para fins de planejamento de unidades

Revista Árvore, Viçosa-MG, v.35, n.2, p.279-288, 2011 
de conservação de proteção integral. Outro aspecto relevante foi a necessidade de adequação do planejamento aos imperativos estabelecidos pelo Sistema Nacional de Unidades de Conservação da Natureza (SNUC), que havia entrado em vigor dois anos antes. O conceito de planejamento contínuo, gradativo, participativo e flexível foi conservado.

Entre as novidades trazidas por esse novo roteiro, podem-se destacar: a) a flexibilização da elaboração do plano de manejo de acordo com as características específicas da unidade; b) a ideia de planejamento com metas a serem atingidas em cenários de curto, médio e longo prazos; e c) as orientações para a confecção de uma versão resumida do plano.

Esse roteiro é hoje referência principal para a elaboração e revisão de planos de manejo para os parques nacionais. Sob sua orientação foram elaborados os planos de manejo do Parque Nacional da Restinga da Jurubatiba e as revisões dos planos dos Parques Nacionais da Tijuca e da Serra dos Órgãos. Apesar de estar em vigor há oito anos, não foi constatada mobilização para revisão do seu conteúdo no curto prazo.

Em relação ao período de vigência, desde o início os planos de manejo foram pensados para serem instrumentos de planejamento temporal, com períodos predeterminados para sua revisão sistemática. Essa característica foi mantida nas legislações que trataram do tema, assim como nos roteiros metodológicos de referência (IBAMA, 1996; IBAMA, 2002). Dessa forma, o caráter dinâmico das transformações a que está sujeita a área protegida e o seu entorno são avaliados com periodicidade, minimizando o engessamento do processo de gestão de longo tempo. Os planos devem ser revisados a cada cinco anos, procurando adequar o zoneamento e os programas à realidade e às necessidades da unidade (IBAMA, 2002). Além disso, o roteiro metodológico de 2002 determina que sua elaboração ou revisão, aprovação e publicação sejam realizadas em um período de oito a 18 meses.

Na prática, contudo, este estudo constatou que para os parques analisados o processo de revisão não operou dentro do cenário determinado. O PARNA da Restinga da Jurubatiba, único entre os parques estudados com plano elaborado sob as recomendações do novo roteiro, levou cerca de 60 meses para ser concluído. Prazo muito superior ao recomendado. Entre os outros parques com planos elaborados sob as instruções de roteiros anteriores, nenhum conseguiu realizar a revisão de seu plano dentro do prazo recomendado. Os PARNAs da Serra dos Órgãos e da Tijuca, únicos que realizaram a revisão, conseguiram concluí-la num prazo de 28 anos para o primeiro e 27 anos para o segundo. As razões para essa situação, recorrentemente indicadas pelos gestores nas entrevistas, apontaram para a questão da falta de recursos financeiros e de equipe técnica nas unidades capazes de executar essa tarefa. Qualquer que seja a explicação, esse fato sublinha uma questão muito mais importante: são os planos de manejo efetivamente instrumento necessário e aplicável à gestão dos parques nacionais?

Os parques nacionais do Estado do Rio de Janeiro compõem amostra que corresponde a quase $10 \%$ do total de parques federais no Brasil. A sua análise pode fortemente sugerir que em boa parte da sua existência os planos de manejo não constituíram ferramenta de uso recorrente para a gestão. Seja porque eles inexistiam ou porque suas versões estavam desatualizadas. Mesmo o PARNA da Restinga da Jurubatiba, apesar de estabelecido em período muito recente (em 1998, os planos de manejo já eram realidade consolidada tanto na legislação quanto metodologicamente), só foi aprovado 10 anos após sua criação, o dobro do tempo previsto pelo seu Decreto de criação.

Outros dois exemplos ilustram bem essa situação. O PARNA do Itatiaia quando foi criado em 1937 tinha área de aproximadamente 12.000 ha, e em 1982 sua área foi ampliada para cerca de 30.000 ha. Como seu plano de manejo foi publicado antes da ampliação e não houve ainda a sua revisão, o zoneamento do parque estava definido somente para a área de 12.000 ha. Esse problema foi em parte solucionado com o PAE em 1994, porém, durante bom tempo, parte do território do parque ficou sem a abordagem gerencial trazida pelo plano de manejo através de seu zoneamento.

Situação semelhante ocorreu com o PARNA da Serra dos Órgãos, que teve a revisão de seu plano de manejo aprovada em 2008 (ICMBIO, 2008b), e quase concomitantemente um Decreto dobrou a extensão de sua área. Nesse caso, como apurado durante a pesquisa, a proposta de ampliação ocorreu como consequência das discussões para revisão do plano de manejo. O que é um aspecto bastante positivo, mas, no entanto, o zoneamento e os programas previstos no novo plano não contemplam as novas áreas incorporadas ao parque. 
Esses exemplos são emblemáticos, mas não significam que o plano de manejo e a gestão do parque devam ficar comprometidos. Pelo contrário, enquanto projeto dinâmico e flexível, conforme definição histórica, os planos de manejo são instrumentos capazes de incorporar novas situações como as descritas anteriormente, que se apresentam na gestão de determinada área a partir das avaliações anuais. O problema é que na prática, como apontaram alguns gestores, isso acaba não ocorrendo na grande maioria dos casos. Em parte porque a elaboração dos planos de manejo conta com a montagem de equipes específicas e a contratação de consultores que se dedicam especificamente a essa tarefa. Uma vez finalizado o plano, cabe à unidade de conservação e à sua equipe de analistas cuidarem da sua implementação. Em muitos casos não existem condições que permitem implementar as ações previstas, quiçá realizar esse tipo de revisão durante a implementação.

No caso dos PARNAs da Tijuca e da Serra dos Órgãos, que tiveram seus planos recentemente revisados e as ações previstas em seus planos anteriores avaliadas, isso pode ser constatado no baixo nível de implementação integral das atividades previstas nos planos originais (26,7\% para o primeiro e $55 \%$ para o segundo) (ICMBio, 2008ab). Esses dados confirmam o mesmo padrão observado em diferentes estudos, em que a efetividade da gestão é recorrentemente mais precária em unidades de conservação sem plano de manejo ou com fraca capacidade de planejamento (BRITO, 2000; PIRES etal., 2000; PRIMOe PELLENS, 2000; FARIA, 2002; LIMA et al., 2005; ZELLER, 2008).

Outra crítica frequente aos planos de manejo é que eles são documentos extensos que dedicam boa parte de seu conteúdo a aspectos descritivos da área e não propriamente aqueles voltados para o seu manejo. Para Dourojeanni (2005), defeito muito comum dos planos de manejo é o grande desequilíbrio que apresentam entre a parte descritiva, desnecessariamente extensa, e a parte analítica e propositiva, muito breve e cheia de lugares comuns de escassa utilidade prática.

Planos de manejo, em geral, contêm centenas de páginas, das quais 50\% a 70\% correspondem à descrição da área e de seu entorno. No caso dos planos analisados neste trabalho, tanto as versões mais antigas quanto, sobretudo, as versões mais recentes são realmente documentos muito extensos. A média geral foi de 571 páginas por plano de manejo, sendo as versões mais antigas as menores (PARNA da Tijuca $=112$ páginas; PARNA da Serra dos Órgãos $=77$ páginas; PARNA do Itatiaia $=206$ páginas; PARNA da Serra da Bocaina = 676 páginas) e as mais recentes maiores (PARNA Restinga da Jurubatiba $=801$ páginas; revisão PARNA da Serra dos Órgãos = 702 páginas; e revisão PARNA da Tijuca $=1.300$ páginas). Em todos os casos, os planos analisados usaram a maior parte do conteúdo produzido com informações descritivas da área e do contexto (internacional, nacional e regional), em que o parque estava inserido. Apenas fração menor dos documentos analisados correspondeu às informações sobre o zoneamento e as proposições de manejo.

A inclusão de informações descritivas são elementos necessários no plano de manejo, conforme recomenda o roteiro metodológico, pois elas servem de base para a construção do zoneamento e dos programas de ação. Contudo, elas não podem se constituir no plano de manejo em si. Em geral, a análise do conteúdo dos planos de manejo para os parques no Rio de Janeiro indicou que os planos mais recentes, feitos sob a orientação do novo roteiro (IBAMA, 2002), tenderam a apresentar melhor o planejamento de execução das atividades com a definição de indicadores para o monitoramento e avaliação. Entretanto, não foram encontrados trabalhos na literatura corrente, sejam acadêmicos ou relatórios institucionais, que tratem da efetividade das ações propostas nos planos nem de suas avaliações sistemáticas. Apesar de estarem previstas no roteiro, as avaliações anuais da implementação do plano de manejo não estão disponíveis nos parques estudados e nem foram objeto ainda de análise mais detalhada.

O financiamento para a elaboração e revisão de planos de manejos representa efetivamente sério problema a ser superado. Isso refletiu claramente na baixa capacidade que os parques nacionais do Rio de Janeiro apresentaram para cumprimento dos prazos estabelecidos para elaboração e revisão dos planos. Para Silva (2005), há tendência para reversão desse cenário em médio prazo, tendo em vista que a partir de 2005 US\$ 17 milhões estariam disponíveis para a criação de novas unidades de conservação e a elaboração e implementação de planos de manejo. Somado a esses valores, os recursos oriundos da compensação estabelecida pelo SNUC serão também importante fonte para financiamento do manejo em unidades de conservação. Até 2005, esses recursos já tinham atingido cerca de US\$ 67 milhões, sendo parcela desse montante aplicada na confecção de planos de manejo (SILVA, 2005).

Revista Árvore, Viçosa-MG, v.35, n.2, p.279-288, 2011 


\section{CONCLUSÃO}

A gestão de áreas protegidas é um processo contínuo e necessário para que efetivamente se cumpram os objetivos estabelecidos para a sua proteção. Contudo, ele demanda planejamento, organização e recursos humanos e financeiros. Os planos de manejo foram instituídos no Brasil no final da década de 1970, visando estabelecer uma abordagem metodológica objetiva para a gestão, inicialmente, de parques nacionais, mas, nos anos posteriores, também para outras categorias de unidades de conservação.

Conclui-se com este trabalho que, apesar do aperfeiçoamento dessa ferramenta ao longo dos anos, expresso nos distintos roteiros metodológicos de referência, sua implementação no Brasil continua precária, especialmente nos parques nacionais do Estado do Rio de Janeiro. Foram observadas dificuldades no cumprimento dos seguintes aspectos no plano de manejo: a) elaboração no período de até cinco anos após a criação da UC; b) revisão a cada cinco anos; c) elaboração e publicação no período máximo de 18 meses; d) execução do planejamento proposto no plano de manejo anterior; e e) adequação de toda a área do parque ao zoneamento ambiental. Tais resultados, associados ao baixo número de parques com plano atualizado, permitem afirmar que os planos de manejo ainda não são plenamente utilizados como instrumento de gestão na área estudada.

O incremento no tamanho das equipes responsáveis pela implementação do plano, assim como mais recursos financeiros, foi frequentemente indicado pelos gestores como solução para esses problemas. Porém, maior objetividade e simplificação no processo de elaboração dos planos, com mais foco no planejamento das ações de gestão e sua avaliação periódica, revelaram-se um problema maior ou de magnitude semelhante a ser enfrentado no manejo dos parques nacionais do Rio de Janeiro.

\section{AGRADECIMENTOS}

As chefes e gestores de todos os parques nacionais estudados neste trabalho, pela contribuição para o desenvolvimento desta pesquisa; à Dra. Angela Tresinari, pelas informações; ao CNPq, pelo financiamento no âmbito do INCT-Políticas Públicas, Estratégias e Desenvolvimento; e aos dois revisores anônimos da revista, pelas sugestões.

Revista Árvore, Viçosa-MG, v.35, n.2, p.279-288, 2011

\section{REFERÊNCIAS}

BARDIN, L. Análise de conteúdo. Lisboa: Edições 70, 1977. 223p.

BRASIL. Decreto $n^{\circ}$ 23.793, de 23 de janeiro de 1934. Aprova o Código Florestal, 1934. Acesso em dezembro de 2008. Disponível em: http://www.planalto.gov.br/ ccivil_03/Decreto/ 1930-1949/D23793.htm

BRASIL. Decreto $n^{\circ}$ 84.017, de 21 de setembro de 1979. Aprova o regulamento dos Parques Nacionais Brasileiros, 1979. Acesso em dezembro de 2008. Disponível em: http:// www.planalto.gov.br/ccivil_03/decreto/1970-1979/ D84017.htm

BRASIL. Decreto de 29 de abril de 1998. Cria o Parque Nacional da Restinga de Jurubatiba, 1998. Acesso em dezembro de 2008. Disponível em: https://www.planalto.gov.br/ccivil_03/dnn/ anterior\%20a\%202000/1998/dnn6730.htm

BRASIL. Lei no $\mathbf{9 . 9 8 5}$, de 18 de julho de 2000. Institui o Sistema Nacional de Unidade de Conservação da Natureza, 2000. Acesso em dezembro de 2008. Disponível em: http:// www.planalto.gov.br/ccivil_03/Leis/L9985.htm

BRASIL. Base da Legislação Federal. 2008. Acesso em dezembro de 2008. Disponível em: https:/ /legislacao.planalto.gov.br/legisla/legislacao.nsf fraWeb?OpenFrameSet\&Frame $=$ frmWeb2\&Src $=/$ legisla legislacao.nsf\%2FFrmConsultaWeb1\%3F Open Form\% 26Auto Framed>

BRITO, M. A. Avaliação do Nível de Implementação das Unidades de Conservação do Estado do Mato Grosso. In: CONGRESSO BRASILEIRO DE UNIDADES DE CONSERVAÇÃO, 2., 2000, Campo Grande. Anais... Campo Grande: 2000. v.2. p.645-653.

BROOKS, T. M. et al. Coverage provided by the global protected area system: is it enough? BioScience, v.53, n.9, p.1081-1091, 2003.

DOUROJEANNI, M. Planos de Manejo. Jornal O ECO, 02 ago., 2005.

ERVIN, J. Protected areas assessements in perspective. BioScience, v.53, n.9, p.819-822, 2003. 
FARIA, H. H. Estado da Gestão de Três Unidades de Conservação do Estado de São Paulo. In: CONGRESSO BRASILEIRO DE UNIDADES DE CONSERVAÇÃO, 3., 2002, Fortaleza. Anais... Fortaleza: 2002. p.289-303.

HOCKINGS, M. Systems for assessing the effectiveness of management in protected areas. BioScience, v.53, n.9, p.823-832, 2003.

HOROWITZ, C. O. Processo de Planejamento e Manejo de Unidades de Conservação Federais de Proteção Integral: O Caso do Parque Nacional de Brasília. In: CONGRESSO BRASILEIRO DE UNIDADES DE CONSERVAÇÃO, 2., 2000, Campo Grande. Anais... . Campo Grande: 2000. p.462-471.

IBDF/FBCN. Plano de Manejo do Parque Nacional da Serra dos Órgãos. Brasília: 1980. 128p.

IBDF/FBCN. Plano de Manejo do Parque Nacional da Tijuca. Brasília: 1981.115p.

IBDF/FBCN. Plano de Manejo do Parque Nacional do Itatiaia. Brasília: 1982. 207p.

IBAMA. Plano de Ação Emergencial para o Parque Nacional da Serra dos Órgãos. Brasília: 1994a. 111p.

IBAMA. Plano de Ação Emergencial para o Parque Nacional do Itatiaia. Brasília: 1994b. 91p.

IBAMA. Roteiro Metodológico para a Elaboração de Planos de Ação Emergencial das Unidades de Conservação de Uso Indireto. $3^{\text {a }}$ versão. Brasília: 1995. 24p.

IBAMA. Roteiro Metodológico para a Elaboração de Plano de Manejo em Unidades de Conservação de Uso Indireto. Brasília: IBAMA/GTZ, 1996.

IBAMA. Roteiro metodológico de planejamento - parques nacionais, reserva biológica, estação ecológica. Diretoria de Ecossistemas/DIREC do IBAMA, 2002.

IBAMA. Siucweb. 2008. Acesso em dezembro de 2008. Disponível em: <http://www.ibama.gov.br/ siucweb>
ICMBIO. Plano de Manejo do Parque Nacional da Tijuca. Brasília: 2008a. 1365p.

ICMBIO. Plano de Manejo do Parque Nacional da Serra dos Órgãos. Brasília: 2008b. 665p.

ICMBIO. Plano de Manejo do Parque Nacional da Restinga da Jurubatiba. Brasília: 2008c. 801p.

LIMA, G.S.; RIBEIRO, G. A.; GONÇALVES, W. Avaliação da Efetividade de Manejo das Unidades de Conservação de Proteção Integral em Minas Gerais. Revista Árvore, v.29, n.4, p.647-653, 2005

LOVEJOY, T. E. Protected areas: a prism for a changing world. Trends in Ecology and Evolution, v.21, n.3, p.329-333, 2006.

BRASIL. Ministério da Agricultura. Cadastro Nacional de Unidades de Conservação. Disponível em <http://sistemas.mma.gov.br/ portalcnuc> Acesso em: 10 de dez. 2008.

MEDEIROS, R.; IRVING, M.; GARAY, I. A proteção da natureza no Brasil: evolução e conflitos de um modelo em construção.

Revista Desenvolvimento Econômico, n.9, p.83-93, 2004.

MEDEIROS, R. Evolução das tipologias e categorias de áreas protegidas no Brasil. Revista Ambiente e Sociedade, v.9, n.1, p.41-64, 2006.

MEDEIROS, R.; GARAY, I. Singularidades do sistema de áreas protegidas no Brasil e sua importância para a conservação da biodiversidade e o uso sustentável de seus componentes. In: GARAY, I.; BECKER, B. (Orgs.). Dimensões humanas da biodiversidade: o desafio de novas relações sociedade-natureza no século XXI. Petrópolis: 2006. p.159-184.

MILLER, K. Planning National Parks for Ecodevelopment. Methods and Cases from Latin America. Peace Corps. 1989. 680 p.

PÁDUA, J. A. Um Sopro de Destruição Pensamento Político e Crítica Ambiental no Brasil Escravista (1786-1888). Rio de Janeiro: Jorge Zahar, 2003. 318p. 
PARRISH, J.; BRAUN, D. P.; UNNASH, R. S. Are we conserving what we say we are? Measuring ecological integrity within protected areas.

BioScience, v.53, n.9, p.851-860, 2003.

PIRES, A. M. Z. C. R.; SANTOS, J. E.; PIRES, J. S. Conservação da Biodiversidade: Análise da Situação de Unidades de Proteção Integral do Estado de São Paulo. In: CONGRESSO BRASILEIRO DE UNIDADES DE CONSERVAÇÃO, 2., 2000, Campo Grande.

Anais..., Campo Grande: 2000. v.2. p.618-627.

PRIMO, P. B. S.; PELLENS, R. A Situação Atual das Unidades do Estado do Rio de Janeiro. In: II CONGRESSO BRASILEIRO DE UNIDADES DE CONSERVAÇÃO, 2., 2000, Campo Grande.

Anais..., Campo Grande: 2000. v. 2. p. 628-637.

RYLANDS, A. B.; BRANDON, K. Brazilian

protected areas. Conservation Biology, v.19, n.3, p.612-618, 2005.

SANTOS, A. A. O Parque Nacional do Itatiaia. Cadernos FBDS, n.3, p.1-172, 2000.
SILVA, M. The Brazilian Protected Areas Program. Conservation Biology, v.19, n.3, p.608-611, 2005.

SOUZA, P. C.; MARTOS, H. L. Estudo do Uso Público e Análise Ambiental das Trilhas em uma Trilha de Unidade de Conservação de Uso Sustentável na Floresta Nacional de Ipanema, Ipero - SP. Revista Árvore, v.32, n.1, p.91100, 2008.

WETTERBERG, G. B. The concept of protected area system based on pleistocene refuges. In: MILANO, M. S. et al (Orgs). Unidades de Conservação: atualidades e tendências. Curitiba: Fundação O Boticário de Proteção à Natureza, 2004. p.76-91.

ZELLER, R. H. Aplicabilidade dos Planos de Manejo de oito Parques Nacionais do Sul e Sudeste do Brasil. 2008. 166p. Dissertação (Mestrado em Engenharia Florestal) - Universidade Federal do Paraná, Curitiba, 2008. 